\title{
Publisher's Note: Numerical simulations of stripping effects in high-intensity hydrogen ion linacs [Phys. Rev. ST Accel. Beams 12, 040102 (2009)]
}

\section{J.-P. Carneiro, B. Mustapha, and P. N. Ostroumov} (Received 19 May 2009; published 28 May 2009)

DOI: $10.1103 /$ PhysRevSTAB.12.059902

PACS numbers: 29.27.-a, 41.75.Cn, 99.10.Fg

This paper was published online on 27 April 2009 with errors in Eqs. (1) and (11). Equation (1) should read as " $S(\omega ; T) d \omega=\frac{\hbar}{\pi^{2} c^{3}} \frac{\omega^{3}}{\exp (\hbar \omega / k T)-1} d \omega$ "; and in Eq. (11) the term " $J_{m n}$ " should be replaced by " $J_{n m}$ ". The equations have been corrected as of 20 May 2009. 